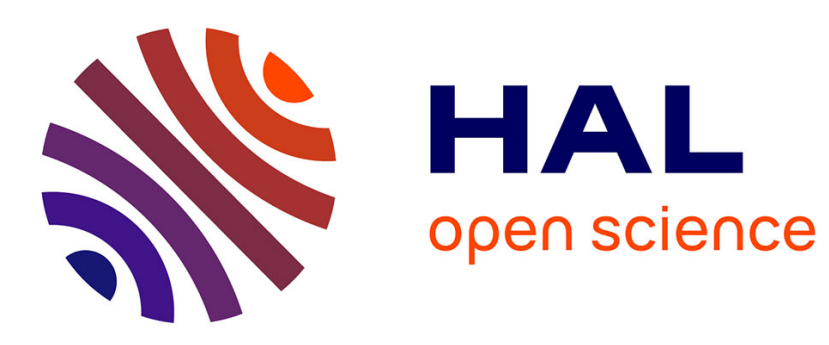

\title{
Proton Radiation Belt Anisotropy as Seen by ICARE-NG Head-A
}

Marine Ruffenach, Sébastien Bourdarie, Julien Mekki, Didier Falguère, Jean-Roch Vaille

\section{- To cite this version:}

Marine Ruffenach, Sébastien Bourdarie, Julien Mekki, Didier Falguère, Jean-Roch Vaille. Proton Radiation Belt Anisotropy as Seen by ICARE-NG Head-A. IEEE Transactions on Nuclear Science, 2019, 66 (7), pp.1753-1760. 10.1109/TNS.2019.2897159 . hal-02315268

\section{HAL Id: hal-02315268 \\ https://hal.science/hal-02315268}

Submitted on 14 Oct 2019

HAL is a multi-disciplinary open access archive for the deposit and dissemination of scientific research documents, whether they are published or not. The documents may come from teaching and research institutions in France or abroad, or from public or private research centers.
L'archive ouverte pluridisciplinaire HAL, est destinée au dépôt et à la diffusion de documents scientifiques de niveau recherche, publiés ou non, émanant des établissements d'enseignement et de recherche français ou étrangers, des laboratoires publics ou privés. 


\title{
Proton radiation belts anisotropy as seen by ICARE-NG Head-A
}

\author{
M. Ruffenach, S. Bourdarie, J. Mekki, D. Falguère, and J. R. Vaillé
}

\begin{abstract}
The ICARE-NG instrument onboard the Argentinian satellite SAC-D detected much more protons during descending orbits (when latitude decreases) than for ascending orbits (increasing latitudes). In this paper we will investigate on the anisotropy seen by the ICARE-NG Head-A for protons in coincidence mode from Monte-Carlo simulations performed with GEANT4. Our simulations show that the difference in the fluxes observed during ascending and descending orbits comes from the fact that the instrument observed trapped protons or not on each point of the orbits as a result of the instrument and satellite orientations. In addition, we show in this paper that the measurements performed by ICARE-NG can be used in conjunction with our GEANT4 simulations to study the anisotropy of trapped protons, i.e. their distribution relative to their equatorial pitch-angle.
\end{abstract}

Index Terms-Anisotropy, Geant4, Monte-Carlo simulations, proton, radiation belts, radiation monitor, response function, space environment.

\section{INTRODUCTION}

$\mathrm{S}$ INCE their discovery in 1958 by Explorer and Pioneer probes, the Earth's radiation belts are widely studied especially because kinetic energies of particles are very high and may cause damages on human bodies and on spacecraft, sometimes leading to their loss [1] [2]. In order to better understand radiation belts, monitors are implemented onboard satellites to give more information about particles trapped in radiation belts. Several studies have been done to investigate anisotropy in the inner belt. In particular, Siegl [3] uses a combination of the AP8 omnidirectional flux model [4] [5] with the Badhwar-Konradi model [6] to investigate anisotropy for SREM [7] coincidence channels. The shape parameter of the Badhwar-Konradi model is calculated by fitting the distribution of the flux given by the model to the AP8 fluxes of $20 \mathrm{MeV}$ trapped protons. Based on this model we will calculate the shape parameter out of fluxes recorded by the ICARE-NG Head-A (and not using AP8) for $18.6 \mathrm{MeV}$ trapped protons to compare with results obtained by Siegl, and

Manuscript submitted December 20, 2018.

This work was conducted as part of an ONERA internship and an ONERA / CNES thesis.

M. Ruffenach, S. Bourdarie and D. Falguère are with ONERA The French aerospace lab / Département Physique Instrumentation Environnement et Espace, 2 Av. Edouard Belin, FR-31055 Toulouse Cedex 4, France (telephone: +33562 2525 68, e-mail: Marine.Ruffenach@onera.fr).

J.R. Vaillé is with the Université de Montpellier, IES - UMR UM/CNRS 5214, 860 Rue de St Priest, Bat. 5, F-34097 Montpellier, France.

J. Mekki is with CNES, 18 av. E. Belin 31401 Toulouse, France. for $81 \mathrm{MeV}$ trapped protons to evaluate the energy dependence of the anisotropy. Other models will be used in this paper to do more comparisons.

In section II, a brief presentation of the ICARE-NG instrument will be given. In section III conditions of GEANT4 [8] [9] [10] simulations are presented with the description of the particles' source and the establishment of the protons response function in coincidence mode of ICARE-NG HeadA. Section IV focuses on GEANT4 simulations for the monitor on-board the SAC-D satellite. In particular, matrices providing the number of particles which can be measured according to their equatorial pitch-angle will be established for several satellite's locations and orbit type (ascending and descending orbit). Finally, in section $\mathrm{V}$ we will establish a model describing the anisotropy for $18.6 \mathrm{MeV}$ and $81 \mathrm{MeV}$ energies protons, respectively the lowest and the highest energies not contaminated by electrons in coincidence mode for the ICARE-NG Head-A.

\section{ICARE-NG INSTRUMENT}

This paper focuses on the CNES ICARE-NG instrument onboard the low Earth orbiting Argentinian satellite SAC-D which flew between June 10, 2011 and June 8, 2015. The complete description of the satellite and instrument can be found in [11] and in [12]. The satellite was on a sunsynchronous orbit at a mean altitude of $650 \mathrm{~km}$ and an orbit inclination of $98.01^{\circ}$. The ICARE-NG instrument consists of three detector heads (A, B, and C). Both sensors A and C have a pair of $500 \mu \mathrm{m}$ thick silicon diodes such that a coincidence and an anti-coincidence acquisition mode are available. These heads are set to measure protons and electrons. The Head-B is composed of a single $700 \mu \mathrm{m}$ thick silicon diode to measure electrons. Particle fluxes for electrons from $250 \mathrm{keV}$ to $3.2 \mathrm{MeV}$ and for protons from $12.8 \mathrm{MeV}$ to $190 \mathrm{MeV}$ are obtained. SAC-D was a spin-stabilized satellite, thus ICARENG always pointed to the same direction which was $45^{\circ}$ from the zenith and from the satellite velocity vector (see [12] and section IV for more details). When the satellite was flying on descending orbits (decreasing latitudes), the ICARE-NG monitor recorded fluxes higher than the ones for ascending orbits (increasing latitudes). An illustration of these measurements is shown in Fig. 1 for protons of $65 \mathrm{MeV}$ detected in coincidence mode with the Head-A during January, 2015. This is attributed to the anisotropy of the environment. 


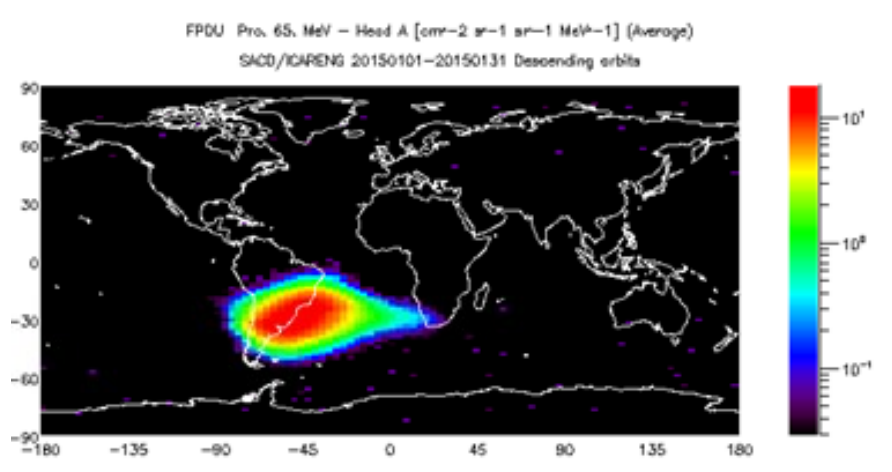

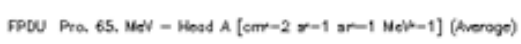
SUCD/1CAMON 20150101-20150131 Ascending orbits

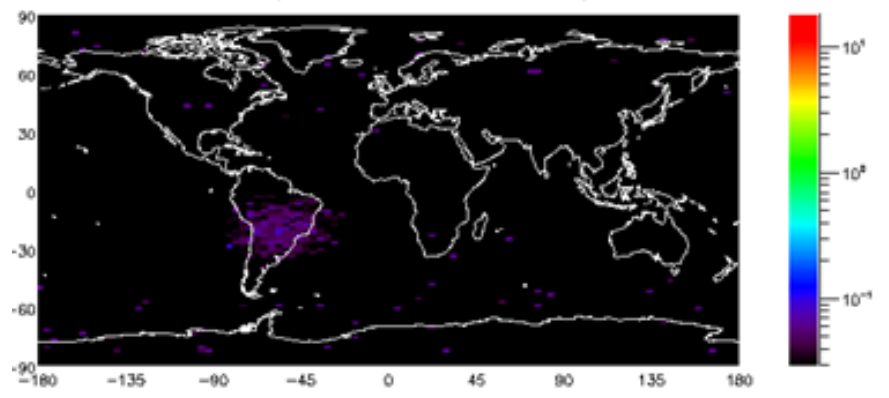

Fig. 1. Fluxes recorded by the Head-A during January 2015 for protons of $65 \mathrm{MeV}$. Top: Descending orbit. Bottom: Ascending orbit.

In this paper we focus on the Head-A of ICARE-NG in coincidence mode for which deposited energy is discretized in 128 channels from $0 \mathrm{MeV}$ to $9.51 \mathrm{MeV}$. To improve the signal to noise ratio original channels may be summed up when level 2 data are produced (Table I).

TABLE 1

ICARE-NG ENERGY DEPOSITION CHANNELS AND DETECTED PROTON ENERGY

\begin{tabular}{cc}
\hline Channels & Energy $(\mathrm{MeV})$ \\
\hline A coincidence 50-78 & 18.6 \\
A coincidence 9 & 115 \\
A coincidence 10 & 100 \\
A coincidence 11 & 90 \\
A coincidence 12 & 81 \\
A coincidence 13 & 74 \\
A coincidence 14 & 69 \\
A coincidence 15 & 65 \\
A coincidence 16 & 63 \\
\hline \hline
\end{tabular}

\section{GEANT4 SimUlations For THE ICARE-NG HEAD-A}

Response function of protons in coincidence mode of the Head-A is computing from a Monte-Carlo simulation based on the GEANT4 toolkit. The geometry of the Head-A was drawn and translated into GEANT-4 compatible mesh using FASTRAD Software [13] [14]. In the simulations described in sections III and IV, incident energies are defined from $14 \mathrm{MeV}$ to $150 \mathrm{MeV}$ by step of $2 \mathrm{MeV}$ to cover the full energy range seen by the Head-A. When very good statistical results are required, the Monte-Carlo simulation is performed with 10 million tries otherwise two million tries are assumed to be good enough. In these simulations a particle source with an isotropic distribution is assumed, thus we consider a spherical source with a radius equals to $3.9 \mathrm{~cm}$ and a cosine-law angular distribution for velocity vector directions [15]. With this source, particles come from all around the Head-A.

To have information about privileged detected particles taking into account the shielding of the Head-A surrounding diodes we calculate the geometric factor. The geometric factor $G E F$ is obtained from the ratio between the number of particles detected per channel over the total number of particles launched by incident energy, expression given in (1) and calculated using [15] and [16].

$$
G E F=4 \pi^{2} R^{2} \frac{N_{d e t}}{N_{\text {Total }}}
$$

where $R$ is the radius of the source.

Response function to protons in coincidence mode is shown on Fig. 2.

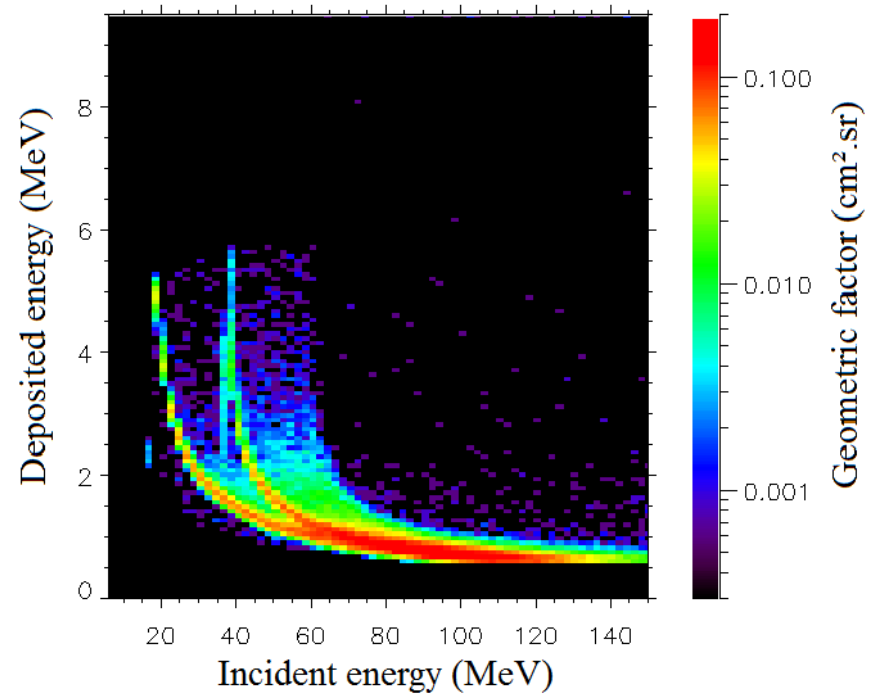

Fig. 2. Geometric factor $\left(\mathrm{cm}^{2}\right.$.sr) in an incident energy (X-axis) - deposited energy (Y-axis) map for ICARE-NG Head-A on-board SAC-D in coincidence mode.

In this response function two branches can be seen, the first one is attributed to particles coming from the main entrance of the detector, and the second one is attributed to penetrating particles crossing the shielding. This is the response function for protons in coincidence mode for particles coming from all around the Head-A without taking into account the satellite shielding and any anisotropy of the proton environment. In the following section we will take into consideration the location and orientation of the satellite to calculate which particles' pitch-angle are reaching the detectors.

\section{The INFLUENCE OF THE SATELLite's Position ON PRIVILEGED DIRECTIONS OF INCOMING PARTICLES}

Since the anisotropy is expected to be more pronounced in coincidence mode than in anticoincidence mode we focus here on the analysis of the response function in coincidence mode of the Head-A. Indeed, to be able to see the impact of the anisotropy we must have a narrow field of view otherwise any 
anisotropy would be smoothed out because of the angle averaging for a large field of view (in the case of anticoincidence for example).

According to the conservation of the first adiabatic invariant along a drift shell we have:

$$
\frac{1}{B_{m}}=\frac{\sin ^{2}\left(\alpha_{e q}\right)}{B_{e q}}=\frac{\sin ^{2}\left(\alpha_{\text {local }}\right)}{B_{\text {local }}}
$$

with $\alpha_{e q}$ the equatorial pitch-angle and $\alpha_{\text {local }}$ the pitch-angle of the particle at the considered position. $B_{\text {local }}, B_{m}$ and $B_{e q}$ are respectively the magnetic field magnitude at the considered point, the magnetic field magnitude at the mirror point, and the magnetic field amplitude at the magnetic equator. We will use this equation in particular to convert local pitch-angles into equatorial pitch-angles.

In order to study the anisotropy it is necessary to know particles velocity vector information and the magnetic field vector for each location along the spacecraft orbit. These values have to be expressed in the same frame to calculate the pitch-angle. Particles coordinates and their velocity vector provided by GEANT4 are expressed in the ICARE-NG coordinate system while the magnetic field vector coordinates are expressed in the geographic system. Knowing the position of ICARE-NG on the satellite [17], and knowing the spacecraft orientation with respect to the Earth, it is then possible to express particle coordinates in the geographic coordinate system to compute the local pitch-angle of incident particle called $\alpha_{\text {local }}$, impinging the Head-A of ICARE-NG at each orbit location.

The ICARE-NG sensor is part of CARMEN equipment [18] on-board SAC-D. The location of ICARE-NG on the satellite is illustrated on Fig. 3.

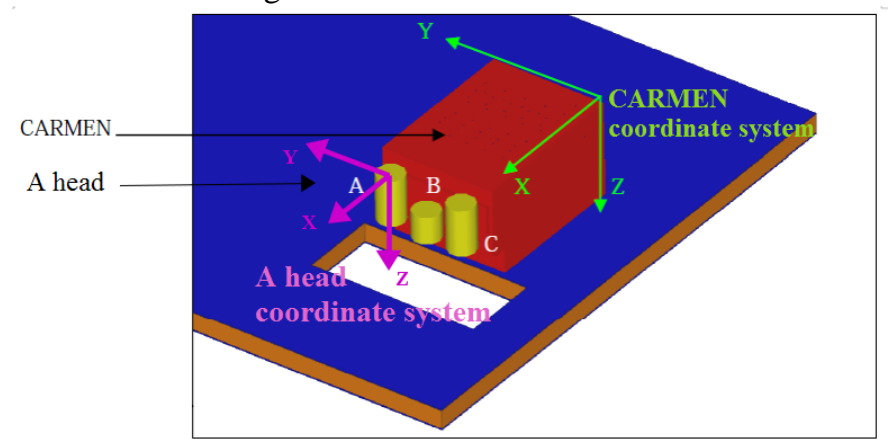

Fig. 3. CARMEN and Head-A coordinate systems.

The origins of the two coordinate systems are separated by a few dozen centimeters so we can neglect Earth magnetic field variations on such distance. In this respect we assume that the CARMEN coordinate system is equivalent to that of the Head-A. Then, the geometry of the SAC-D satellite and the orientation of the ICARE-NG instrument according to the satellite axis are shown in Fig. 4.

To convert from the detector coordinate system to the satellite coordinate system we need to rotate the coordinates by an angle of $-3 \pi / 4$ around the $\mathrm{Y}$-axis. So, the relation to express a vector in the satellite coordinate system from the detector coordinate system is given by (3).

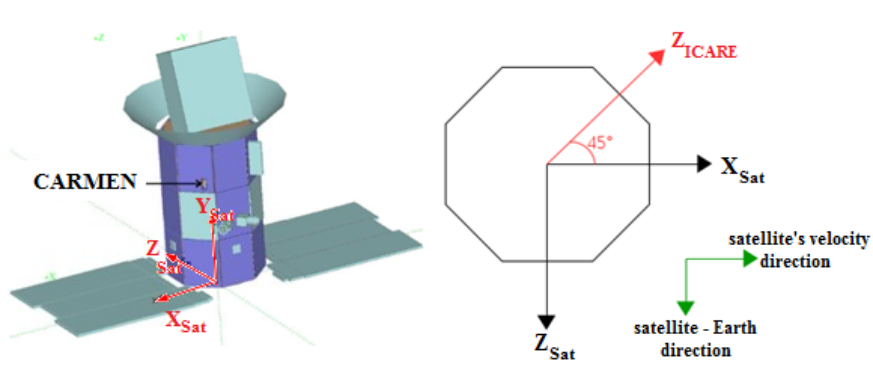

Fig. 4. Left: Geometry of the SAC-D satellite. Right: Orientation of the ICARE-NG axis in the satellite frame.

$$
\left(\begin{array}{l}
x_{\text {sat }} \\
y_{\text {sat }} \\
z_{\text {sat }}
\end{array}\right)=\left(\begin{array}{ccc}
-\frac{1}{\sqrt{2}} & 0 & \frac{1}{\sqrt{2}} \\
0 & 1 & 0 \\
-\frac{1}{\sqrt{2}} & 0 & -\frac{1}{\sqrt{2}}
\end{array}\right)\left(\begin{array}{l}
x_{\text {det }} \\
y_{\text {det }} \\
z_{\text {det }}
\end{array}\right)
$$

Now, we have to convert satellite coordinates into geographic coordinates. The velocity of the satellite being along the $\mathrm{X}$-axis of the satellite coordinate system, the Z-axis of the satellite always points toward the Earth and the Y-axis completes this trihedral. For all the satellite locations along its orbit, its geodesic coordinates (longitude, latitude, altitude) are known and can be converted into geographic coordinates. From these geographic coordinates we can calculate the velocity of the satellite using two consecutive points of the orbit and the Earth-Satellite direction in the geographic system. After normalization, it gives us the conversion of the $\mathrm{X}$-axis and the $\mathrm{Z}$-axis from the satellite coordinate system to the geographic system. The $\mathrm{Y}$-axis projected in the geographic coordinate system completes this trihedral. Having the velocity of particles $\overrightarrow{V_{G E O}}$ and the magnetic field $\overrightarrow{B_{\text {local }}}$ in the same coordinate system, it is straightforward to calculate the local pitch-angle using the scalar product given in (4).

$$
\overrightarrow{B_{\text {local }}} \cdot \overrightarrow{V_{G E O}}=\left\|\overrightarrow{B_{\text {local }}}\right\| \cdot\left\|\overrightarrow{V_{G E O}}\right\| \cdot \cos \left(\alpha_{\text {local }}\right)
$$

Due to the Earth magnetic field properties, eccentric, tilted field with respect to geographic coordinates, SAC-D will encounter a given field line, at different $\frac{B_{\text {local }}}{B_{\text {eq }}}$ at different longitudes. This is to say that the maximum equatorial pitchangle ICARE-NG can measure on a given field line depends on the longitude at which it will cross this given field line. The maximum equatorial pitch-angle SAC-D/ICARE-NG can measure as a function of the longitude is illustrated on Fig. 5 for $\mathrm{L}=1.185$ at an altitude of $664 \mathrm{~km}$. These data are extracted from the orbitography of the SAC-D satellite.

In order to assess the anisotropy of trapped protons on a given field line, three spacecraft locations are considered (red crosses on Fig. 5). For each position, two configurations are possible: ascending orbit and descending orbit.

The equatorial pitch-angle which defines the loss-cone is such that the particle has a mirror point at an altitude of $100 \mathrm{~km}$ during its bounce and drift along a given drift shell. 


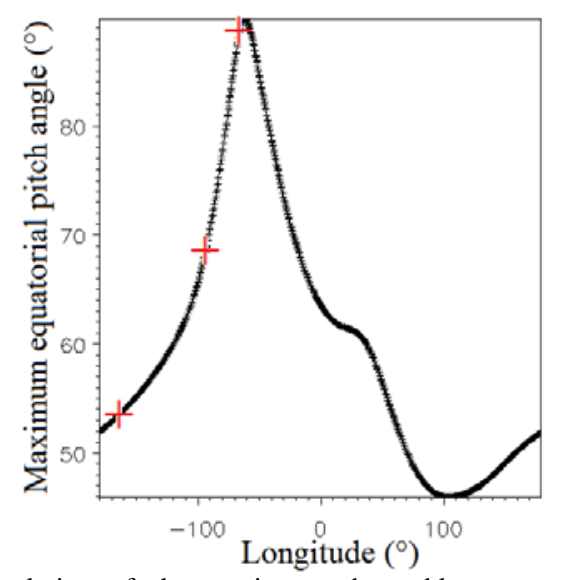

Fig. 5. Evolution of the maximum observable equatorial pitch-angle according to the longitude of SAC-D for a given drift shell with $\mathrm{L}=1.185$ and an altitude equals to $664 \mathrm{~km}$.

Thanks to the equation (2) only the minimal magnetic field value obtained at $100 \mathrm{~km}$ during a drift (limit point for the mirror point beyond which particles precipitate in the upper atmosphere), and the magnetic field value at the magnetic equator are necessary to calculate the loss-cone equatorial pitch-angle. These values are obtained using IRBEM library [19]. This open-source library allows to calculate magnetic coordinates in the Earth's magnetic field and to perform coordinates conversions. For $\mathrm{L}=1.185$ the equatorial pitchangle of the loss-cone is equal to $66.27^{\circ}$. Fig. 6 represents a magnetic field line with a satellite on it. Fig. 7 gives the number of protons reaching the two diodes (among the 2 million particles per bin of $2 \mathrm{MeV}$ being launched) in an incident energy - equatorial pitch-angle map for the three locations represented on Fig. 5 and for ascending and descending orbits. In Fig. 7, the horizontal red line (also represented in red in Fig. 6) indicates the loss cone equatorial pitch-angle (66.27 ${ }^{\circ}$ at $\left.\mathrm{L}=1.185\right)$. Particles with a smaller pitchangle are not trapped and precipitate into the atmosphere.

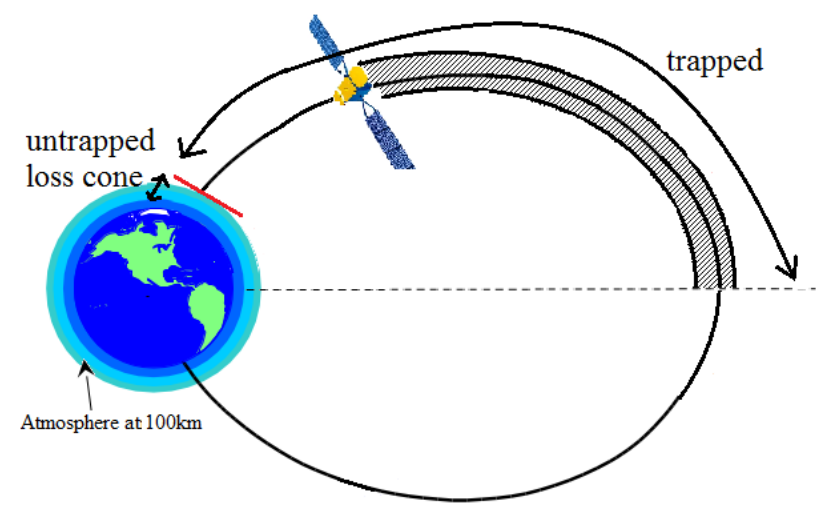

Fig. 6. The satellite on a magnetic field line. The red line represents the limit of the atmosphere where particles precipitate. Hatched area represents particles which are not seen by the satellite since their mirror point is between the satellite and the magnetic equator.
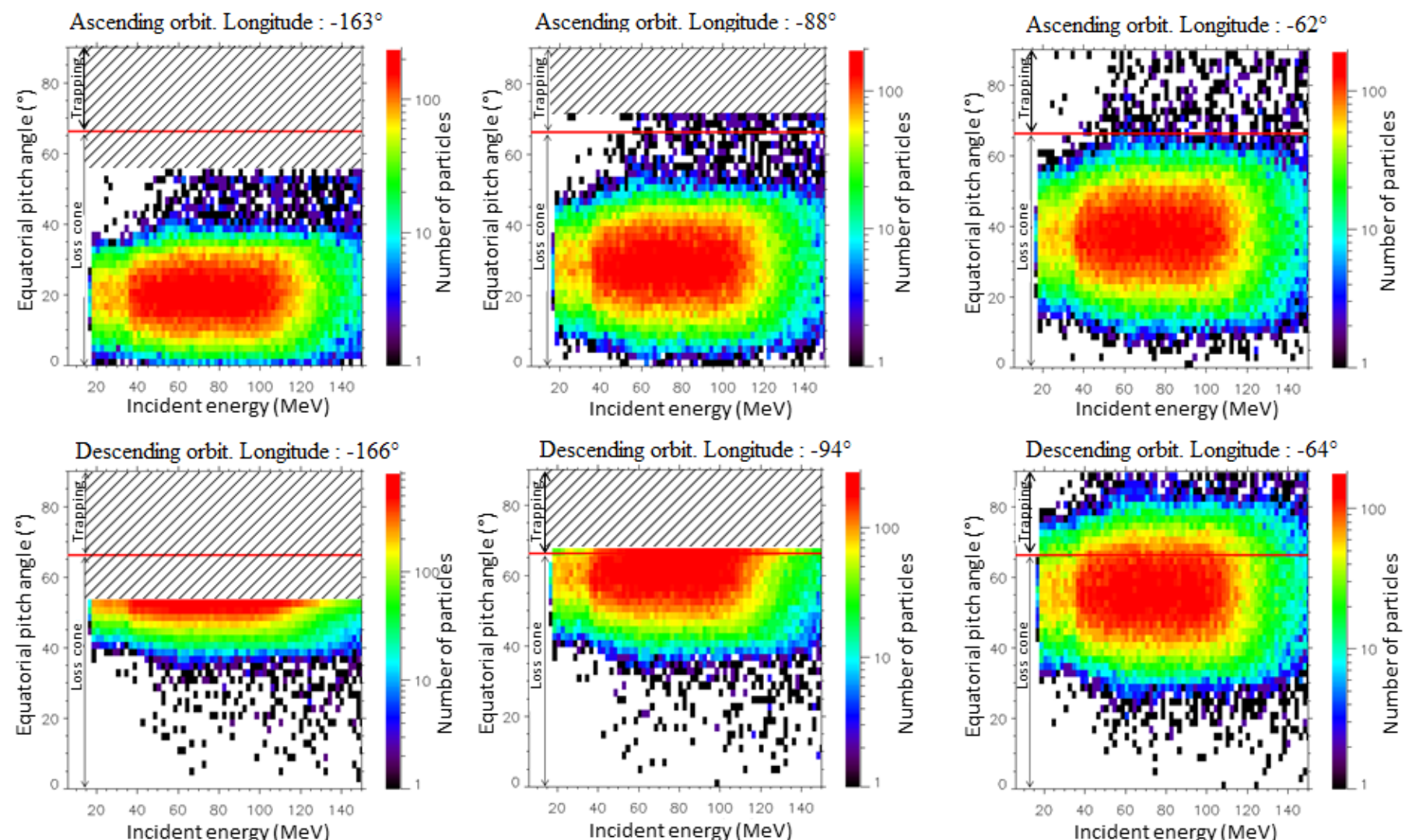

Fig. 7. Number of simulated protons in coincidence mode in an incident energy (X-axis) - equatorial pitch-angle (Y-axis) map. This is for a field line with $\mathrm{L}=$ 1.185 and an altitude equals to 664km. Ascending orbits (top panel) and descending orbits (bottom panel) are classified according to increasing longitudes from left to right. Hatched areas represent particles that cannot be observed because of satellite's position. For each bin of incident energy, two million of particles are simulated. 
Fig. 7 does not represent what the Head-A has actually observed, but it points out what might be the pitch-angles of observed particles. If no particle is observed by the detector, it means that the instrument has no access to trapped particles because of its orientation. The instrument has no access to particles with an equatorial pitch-angle higher than $55^{\circ}$ when the spacecraft is located in the $-165^{\circ}$ longitude range according to matrices on the left, $73^{\circ}$ when the spacecraft is located in the $-90^{\circ}$ longitude range according to matrices on the center, while all equatorial pitch-angles can be observed when the spacecraft is located in the $-63^{\circ}$ longitude range according to matrices in the right panels. These equatorial pitch-angle values are the maximum observable equatorial pitch-angle (cf. Fig. 5), beyond which the sensor does not detect particles regardless of the loss cone. Indeed, this is related to the location of the satellite along a given field line: when it has a magnetic latitude high enough then particles with a high equatorial pitch-angle have a mirror point between the magnetic equator and the satellite (hatched area on Fig. 6). Hatched areas on Fig. 7 represent particles that cannot be observed because of the satellite position along a given field line.

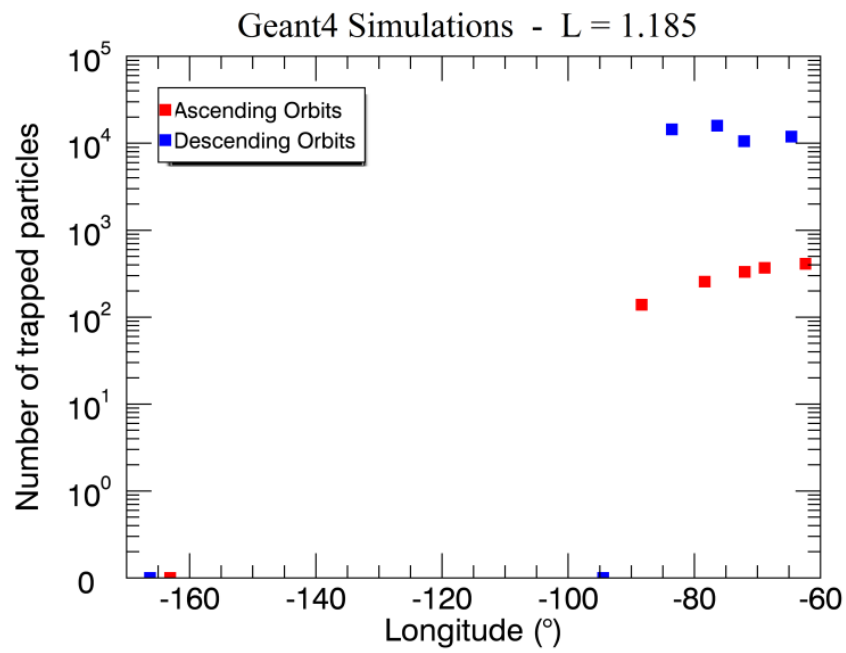

Fig. 8. Evolution of the number of simulated trapped protons summed over all the incident energies (from $14 \mathrm{MeV}$ to $150 \mathrm{MeV}$ ) as a function of longitude for ascending orbits (in red) and descending orbits (in blue).

Fig. 8 gives the number of simulated trapped protons summed over all the incident energies (total number of trapped protons summed from $14 \mathrm{MeV}$ to $150 \mathrm{MeV}$ ) as a function of the spacecraft longitude. As a reminder, Fig. 7 and Fig. 8 are obtained thanks to simulations.

Considering maps at the left in Fig. 7, i.e. when the spacecraft is at a longitude lower than $-95^{\circ}$, the sensor has only access to protons having an equatorial pitch-angle lower than the one defining the loss-cone i.e. all these protons are precipitating into the upper atmosphere. As a result (see Fig. 8 ), when the satellite is at a longitude lower than $-95^{\circ}$, the Head-A has no access to trapped particles so it cannot detect trapped protons at these longitudes. For longitudes greater than $-95^{\circ}$ we can see on Fig. 8 that more and more trapped protons can be measured. Also note that more trapped protons are counted in the case of descending orbits. Maps at the center and on the right of Fig. 7 indicate that the sensor has access to particles with an equatorial pitch-angle higher than the loss cone pitch-angle $\left(66.27^{\circ}\right)$. From the middle maps to the right maps of Fig. 7, the number of trapped particle the sensor has access to is increasing. Also, from top to bottom (middle and right maps of Fig. 7), i.e. increasing or decreasing orbits, the number of trapped protons the sensor has access to is increasing. This is consistent with the results shown in Fig.8 for longitude higher than $-95^{\circ}$.

To summarize, Fig. 7 and Fig. 8 are obtained from GEANT4 simulations. First, we have considered an isotropic environment at the outside of the Head-A. Then, for each location of SAC-D along its orbit we can calculate particles' equatorial pitch-angle impinging the diodes in coincidence mode and find out if the sensor has access to trapped particles or not.

In situ measurements from coincidence channels, like the ones obtained thanks to the ICARE-NG Head-A illustrated in Fig. 1, are well understood with these GEANT4 simulations. They explain qualitatively the difference in the number of detected particles between ascending and descending orbits by highlighting the access to trapped protons or not versus spacecraft location. To fully characterize this anisotropy, in the next section we will perform a comparison between different models to describe the evolution of the unidirectional flux according to the equatorial pitch-angle and the McIlwain L parameter.

\section{ESTIMATING UNIDIRECTIONAL FLUXES}

In this section we will express the unidirectional flux as a function of equatorial pitch-angle according to ICARE-NG measurements during 2013 and local pitch-angle - energy map of this detector. We compare four models of unidirectional fluxes expressed in $\left[\mathrm{cm}^{-2} \mathrm{~s}^{-1} \mathrm{sr}^{-1} \mathrm{MeV}^{-1}\right]$ :

1. Badhwar- Konradi [6] using AP8 model

2. Valot [20] using ICARE-NG data

3. Badhwar-Konradi using ICARE-NG data

4. Roederer [21]

For the second and the third models parameters have to be estimated using in-flight data of ICARE-NG Head-A by comparing counts of particles calculated with these two models from those measured by ICARE-NG. Counts of particles $C$ are calculated thanks to (5):

$$
C=\int_{\alpha_{\text {local }}} \int_{E} \operatorname{flux}\left(E, \alpha_{\text {local }}\right) * \operatorname{Map}\left(E, \alpha_{\text {local }}\right) * d E d \alpha_{\text {local }}
$$

where $\operatorname{Map}\left(E, \alpha_{\text {local }}\right)$ is the geometric factor at a given energy and local pitch-angle. $F l u x\left(E, \alpha_{\text {local }}\right)$ is the unidirectional flux as function of incident energy and local pitch-angle. Unidirectional fluxes for these models are calculated for $L=1.25$ and for two energies, $18.6 \mathrm{MeV}$ and $81 \mathrm{MeV}$.

\section{A. Model 1: Badhwar- Konradi using AP8 model}

This model is the one proposed by NASA to study fluxes at the International Space Station altitude. This distribution is given by (6) for equatorial pitch-angles which are between the 
angle defining the loss-cone and $\frac{\pi}{2}$, otherwise the flux is equal to 0 (in the loss-cone).

$$
j_{B K-A P 8}=K \xi e^{-\beta \xi}
$$

where $\xi=\frac{\sin \left(\alpha_{e q}\right)}{\sqrt{B_{e q}}}-\frac{1}{\sqrt{B_{l c}}}$ [6] with $B_{l c}$ the magnetic field magnitude for a particle having its mirror point on the Earth atmosphere. Magnetic fields magnitudes are expressed in Gauss. The $K$ parameter is a normalization factor calculated so that the integral of differential flux with respect to pitchangles is equal to the omnidirectional flux, but in our case we do not need to calculate this parameter since we will compare hereafter normalized unidirectional fluxes. Then, an expression of the $\beta$ shape parameter is given in [22] and used by Siegl in [23]. The shape parameter is calculated for $20 \mathrm{MeV}$ trapped protons using the AP8 model as a function of the Mcllwain L parameter as shown in (7).

$$
\beta[\sqrt{G}]=\frac{1}{0.13164-8.8674 \ln (L)}
$$

\section{B. Model 2: Valot model using ICARE-NG data}

The Valot model is given in (8) where $n$ and $j_{0}$ have to be estimated, and where $\alpha_{l c . e q}$ is the equatorial pitch-angle defining the loss-cone (mirror point at $100 \mathrm{~km}$ of altitude in the upper atmosphere).

$$
j_{\text {Valot }}=j_{0}\left(\sin \left(\alpha_{e q}\right)^{n}-\sin \left(\alpha_{c p . e q}\right)^{n}\right)
$$

Count of particles are calculated using local pitch-angles while the Valot model uses equatorial pitch-angles. Moreover, Monte-Carlo simulations gives us the number of particles in an incident energy - local pitch-angle map that we call number $\left(E, \alpha_{\text {local }}\right)$. Finally count of particles, expressed in particle per second, is calculated with (9).

$$
\begin{gathered}
C=\int_{\alpha_{\text {local }}} \int_{E} j_{0}\left(\left[\sqrt{\frac{B_{\text {eq }}}{B_{\text {local }}}} \sin \left(\alpha_{\text {local }}\right)\right]^{n}-\sin \left(\alpha_{\text {lc.eq }}\right)^{n}\right) \\
* 4 \pi^{2} R^{2} * \frac{\text { number }\left(E, \alpha_{\text {local }}\right)}{\text { initial number }} \\
* d E d \alpha_{\text {local }}
\end{gathered}
$$

where initial number is the number of particles launched during the Monte-Carlo simulation for a given incident bin in energy. For these simulations we use an initial number equals to ten million of protons per bin of $1 \mathrm{MeV}$ from 14 to $150 \mathrm{MeV}$ to get high fidelity statistical results.

In order to have several points of comparison, we consider all positions of the SAC-D satellite corresponding to the field line with $\mathrm{L}=1.25$ at an altitude of $664 \mathrm{~km}$ during 2013 . We focus on $18.6 \mathrm{MeV}$ protons, so channels with numbers ranging from 50 to 78 for ICARE-NG Head-A, the lowest proton energy for the coincidence mode. For a given channel, i.e. for a given measured incident energy, the count rate is given by (10).

$$
C_{\text {measured }}=\text { flux }_{\text {measured }} * G E F * \Delta E
$$

The $G E F * \Delta E$ factor for $18.6 \mathrm{MeV}$ protons is $0.7 \mathrm{~cm}^{2} . \mathrm{sr} . \mathrm{MeV}$. We have to find $n$ and $j_{0}$ parameters so that counts calculated thanks to (9) are equal to those measured by
ICARE-NG given by (10). We plot counts measured by ICARE-NG as a function of counts calculated thanks to (9), and the objective is to modify $n$ and $j_{0}$ until we obtain a linear plot with a slope close to 1 (equality between counts measured and calculated). We know that for a scatter plot (x,y), the estimation by least squares of the slope of the linear function that best fits these points is given by slope $=\frac{\sum_{i} x_{i} y_{i}}{\sum_{i} x_{i}^{2}}$ and the estimation of the variance of the linear regression is given by $S_{x y}^{2}=\frac{\sum_{i} y_{i}^{2}-\text { slope }^{2} \sum_{i} x_{i}^{2}}{n_{p}-1}$ with $n_{p}$ the number of points. We proceed by dichotomy to find the $n$ parameter which minimizes $S_{x y}^{2}$ and then we adjust the $j_{0}$ parameter to have a slope equals to 1 . Values which give us the best match with measurements of ICARE-NG are $j_{0}=54400 \mathrm{~cm}^{-2} \mathrm{~s}^{-1} \mathrm{sr}^{-1} \mathrm{MeV}^{-1}$ and $n=45$. The result of the minimization process for $\mathrm{E}=18.6 \mathrm{MeV}$ is shown in Fig. 9 in blue.

For ICARE-NG Head-A, the highest energy for protons for the coincidence mode not contaminated by electrons is $81 \mathrm{MeV}$, corresponding to the channel 12 for which the $G E F * \Delta E$ factor is equal to $4.37 \mathrm{~cm}^{2}$. sr. $M e V$. We use the same technic than the one used for the $18.6 \mathrm{MeV}$ energy to find the estimated parameters and we obtain $n=24, j_{0}=$ $15800 \mathrm{~cm}^{-2} \mathrm{~s}^{-1} \mathrm{sr}^{-1} \mathrm{MeV}^{-1}$. The result of the minimization process for $\mathrm{E}=81 \mathrm{MeV}$ is shown in Fig. 10 in blue. Those results indicate that the anisotropy is energy dependent.

\section{Model 3: Badhwar-Konradi model using ICARE-NG data}

The initial formula is the same than the one used for the Badhwar-Konradi model based on AP8.

$$
j_{B K-I C A R E}=k \xi e^{-B \xi}
$$

Instead of using the AP8 model to calculate $B$ we estimate $k$ and $B$ parameters using the same method as used above. We obtain $k=725 \mathrm{~cm}^{-2} \mathrm{~s}^{-1} \mathrm{sr}^{-1} \mathrm{MeV}^{-1} G^{0.5}$ and $B=-10 G^{0.5}$ for an energy of 18.6MeV, and $k=1675 \mathrm{~cm}^{-2} \mathrm{~s}^{-1} \mathrm{sr}^{-1} \mathrm{MeV}^{-1} G^{0.5}$ and $\mathrm{B}=-8 G^{0.5}$ for an energy of $81 \mathrm{MeV}$. The results of the minimization process for 18.6MeV and for $81 \mathrm{MeV}$ are shown in Fig. 9 and in Fig. 10 in red. Again those results indicate that the anisotropy is energy dependent.

\section{Model 4: Roederer using AP8 model}

The Roederer model consists in calculating unidirectional flux from omnidirectional flux given by the AP8 model. The unidirectional flux expression perpendicular to the magnetic field extracted from [21] is given by (12).

$$
j_{\perp}\left(B_{\text {local }}\right)=-\frac{B_{\text {local }}^{3 / 2}}{2 \pi^{2}} \int_{B_{\text {local }}}^{B_{l c}} \frac{d}{d B}\left[\frac{J(B)}{B}\right] \frac{d B}{\sqrt{B-B_{\text {local }}}}
$$

where $\mathrm{J}$ is the omnidirectional flux calculated using the AP8min model. The formula given in (12) allows the calculation of the unidirectional flux as a function of the magnetic field. However, in our case we compute the evolution of the unidirectional flux as a function of the equatorial pitch-angle. The perpendicular unidirectional flux corresponds to a local pitch-angle of $90^{\circ}$ which allows us from 


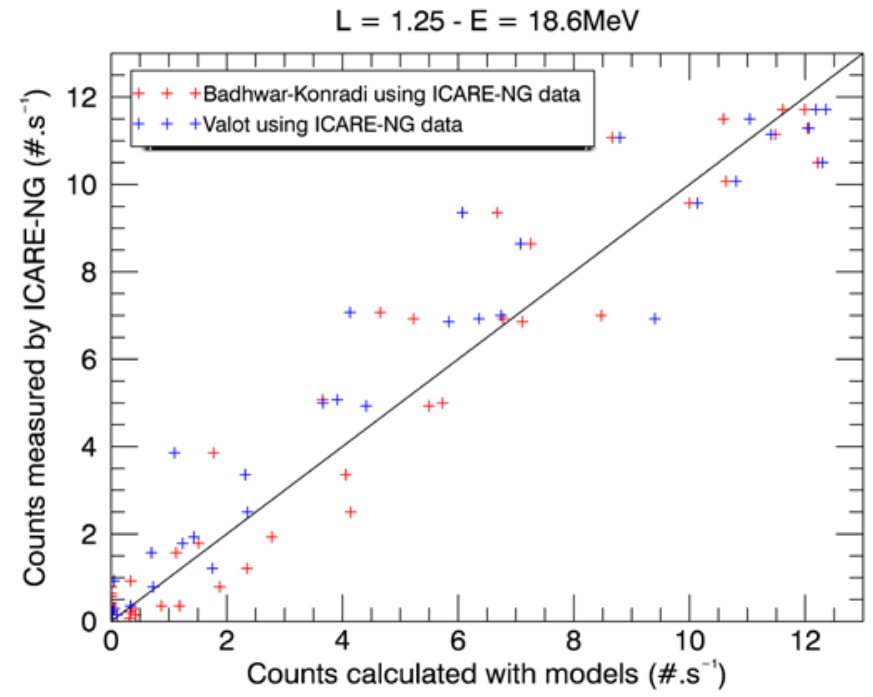

Fig. 9. Result of the minimization process for Valot's model and the Badhwar-Konradi model using ICARE-NG Head-A data for L=1.25 and $\mathrm{E}=18.6 \mathrm{MeV}$ with the ideal straight line in black (if calculated and measured counts were equal). Counts are expressed in number of particles detected per second.

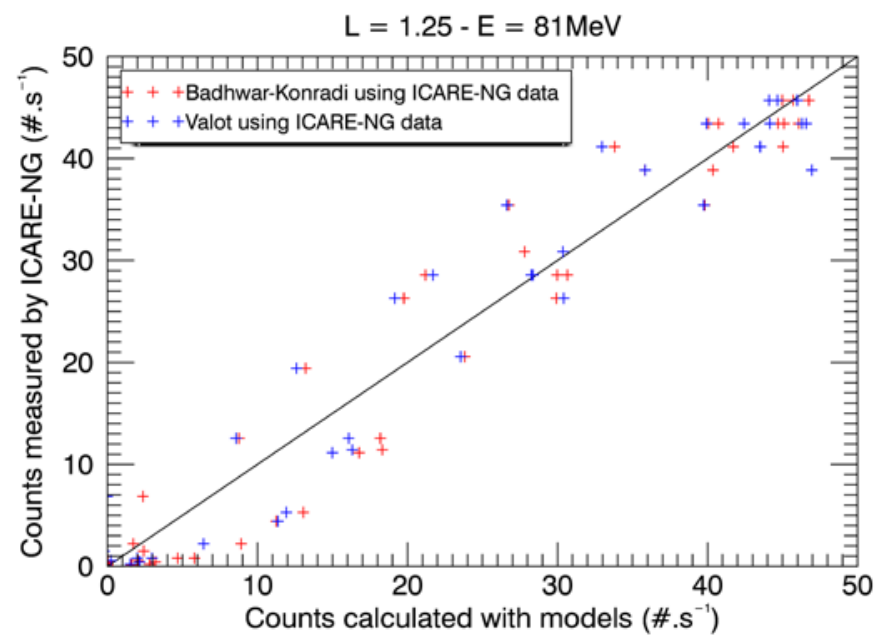

Fig. 10. Result of the minimization process for Valot's model and the Badhwar-Konradi model using ICARE-NG Head-A data for $\mathrm{L}=1.25$ and $\mathrm{E}=81 \mathrm{MeV}$ with the ideal straight line in black. Counts are expressed in number of particles detected per second.

the local magnetic field $B_{\text {local }}$, to calculate the corresponding equatorial pitch-angle using (2).

By comparing results of the minimization process in Fig. 9 and Fig. 10, we can notice that we have almost the same agreement with measurement thanks to the Badhwar-Konradi model based on ICARE-NG measurements than the one obtained thanks to the Valot's model based on ICARE-NG measurements. The four models for an energy of $18.6 \mathrm{MeV}$ are shown in Fig. 11 and they are shown in Fig. 12 for an energy of $81 \mathrm{MeV}$, where fluxes have been normalized to agree at the maximum equatorial pitch-angle $69.68^{\circ}$.

The Valot's model based on ICARE-NG measurements is below all the other models for $18.6 \mathrm{MeV}$, especially for pitchangle close to the loss-cone pitch-angle. The model based on the Roederer method deduces unidirectional fluxes from omnidirectional fluxes, so this model has sharp fluctuations due to the fixed mesh of AP8.

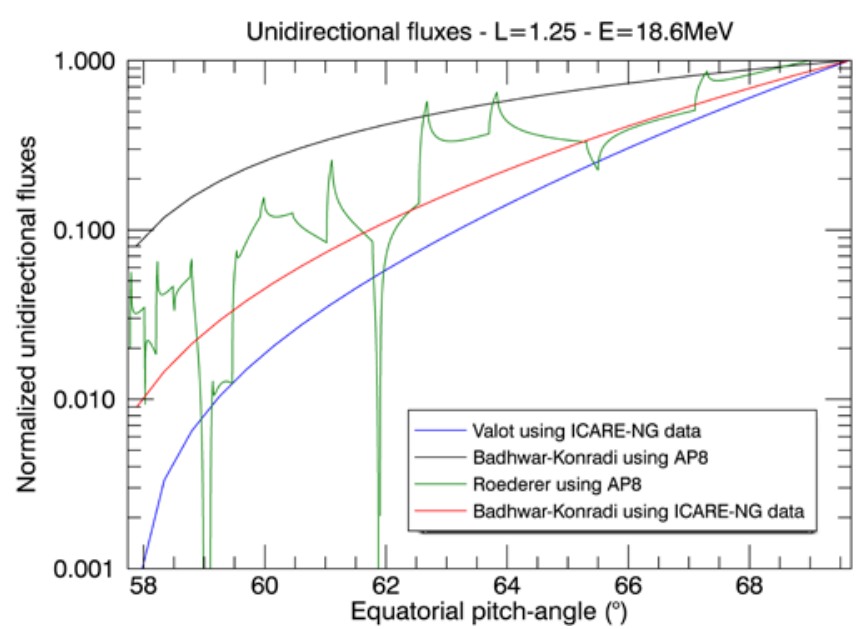

Fig. 11. Evolution of unidirectional flux according to the equatorial pitchangle for different flux models for protons of $18.6 \mathrm{MeV}$ at $\mathrm{L}=1.25$.

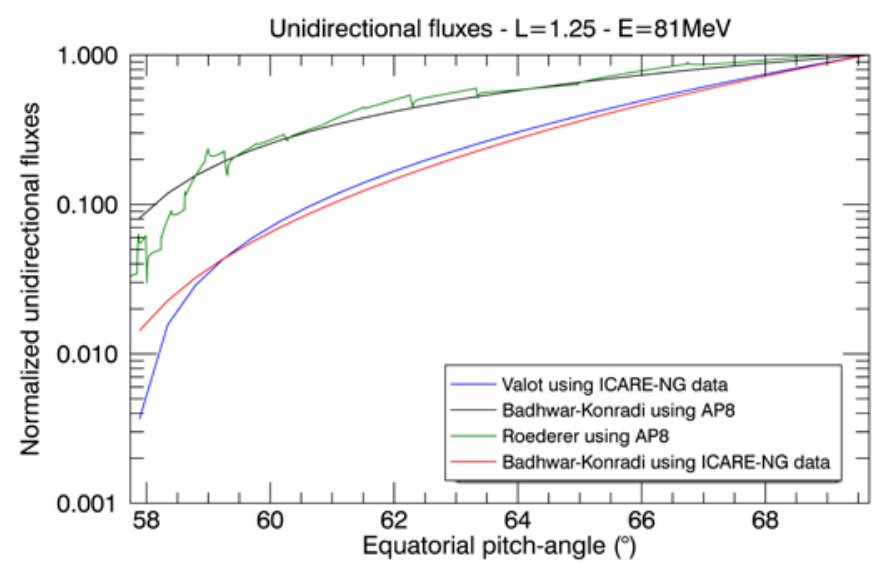

Fig. 12. Evolution of unidirectional flux according to the equatorial pitchangle for different flux models for protons of $81 \mathrm{MeV}$ at $\mathrm{L}=1.25$.

The model based on the Badhwar-Konradi method using AP8 for protons of $20 \mathrm{MeV}$ over-estimates fluxes in comparison to other models. The Badhwar-Konradi method based on ICARE-NG measurements is close to the Roederer model especially for pitch-angles higher than $66^{\circ}$.

Regarding Fig. 12, curves of models based on ICARE-NG Head-A measurements are very close for an energy of $81 \mathrm{MeV}$ except for pitch-angles close to the loss-cone pitch-angle. In general, the Valot's model based on ICARE-NG measurements goes down rapidly for angles near to the losscone pitch-angle since this model is simpler than the other ones. Curves for models based on AP8 have the same variation for $81 \mathrm{MeV}$, although the shape parameter was calculated for $20 \mathrm{MeV}$ protons. The Badhwar-Konradi model based on ICARE-NG measurements seems to be the most appropriate in comparison with the Valot's model to characterize the evolution of the unidirectional flux with the equatorial pitch-angle for protons.

Some studies established that AP8 model overestimates protons fluxes. As indicated in Siegl et al. [3], AP8 overestimates proton fluxes between $\mathrm{L}=1.3$ and $\mathrm{L}=1.6$. Heynderickx et al. [24] have shown that AP8 overestimates fluxes for low energy protons. These overestimations could have an impact on results obtained thanks to the AP8 model. 


\section{CONCLUSION}

Monte-Carlo simulations have been used to investigate anisotropy seen by the Head-A of the ICARE-NG radiation monitor. By combining response function calculated by GEANT4 and real positions of the SAC-D satellite along its orbit we were able to calculate pitch-angles of particles which crossed the two diodes by simulations. Then, by comparing these pitch-angles with the one defining the loss-cone, we were able to say if the head-A sensor in coincidence mode has access to trapped particles or not.

Results in this paper highlight the anisotropy seen by the Head-A of the ICARE-NG instrument. Particles' equatorial pitch-angles impinging the sensor are not the same according to the satellite's location and according to ascending or descending orbits so it allows to characterize the anisotropy seen by ICARE-NG Head-A.

Finally, two models have been implemented to determine unidirectional fluxes out of in-flight measurements of ICARENG Head-A. The study of various models for the evolution of the unidirectional flux according to the equatorial pitch-angle shows that anisotropy depends on the energy of particles and that it is also present at low energy. This work could be used as a complement of specification models including the anisotropy.

All the methodology described in section IV and in section $\mathrm{V}$ is applicable for other missions for which detectors have strong directionality.

\section{REFERENCES}

[1] D. N. Baker, 'The occurrence of operational anomalies in spacecraft and their relationship to space weather', IEEE Transactions on Plasma Science, vol. 28, no. 6, pp. 2007-2016, Dec. 2000.

[2] L. W. Townsend, 'Implications of the space radiation environment for human exploration in deep space', Radiat Prot Dosimetry, vol. 115, no. 1-4, pp. 44-50, Dec. 2005.

[3] M. Siegl, H. D. R. Evans, E. J. Daly, G. Santin, P. J. Nieminen, and P. Bühler, "Inner belt anisotropy investigations based on the standard radiation environment monitor (SREM)," IEEE Trans. Nucl. Sci., vol.57, no. 2, pp. 2017-2023, Apr. 2010.

[4] J. I. Vette, "The NASA/National Space Science Data Center Trapped Radiation Environment Model Program (1964- 1991),” NSSDC/WDCA-R\&S 91-29, 1991.

[5] D. M. Sawyer and J. I. Vette, “AP-8 Trapped Proton Environment for Solar Maximum and Solar Minimum,” NSSDC WDC-A-R\&S 76-06, 1976.

[6] G. D. Badhwar and A. Konradi, 'Conversion of omnidirectional proton fluxes into a pitch angle distribution', Journal of Spacecraft and Rockets, vol. 27, p. 350-352, Jun. 1990.

[7] A. Mohammadzadeh et al., "The ESA standard radiation environment monitor program first results from PROBA-I and INTEGRAL,” IEEE Trans. Nucl. Sci., vol. 50, no. 6, pp. 2272-2277, Dec. 2003.

[8] CERN, "Geant4: A toolkit for the simulation of the passage of particles through matter”. [Online]. Available: http://geant4.cern.ch/.

[9] S. Agostinelli et al., 'Geant4—a simulation toolkit', Nuclear Instruments and Methods in Physics Research Section A: Accelerators, Spectrometers, Detectors and Associated Equipment, vol. 506, no. 3, pp. 250-303, Jul. 2003.

[10] J. Allison et al., “Geant4 developments and applications,” IEEE Trans. Nucl. Sci., vol. 53, no. 1, pp. 270-278, Feb. 2006.

[11] D. Boscher et al., 'In Flight Measurements of Radiation Environment on Board the French Satellite JASON-2', IEEE Transactions on Nuclear Science, vol. 58, no. 3, pp. 916-922, Jun. 2011.
[12] D. Boscher et al., 'In-Flight Measurements of Radiation Environment on Board the Argentinean Satellite SAC-D', IEEE Transactions on Nuclear Science, vol. 61, no. 6, pp. 3395-3400, Dec. 2014.

[13] J. C. Thomas, T. Beutier, P. Pourrouquet, P. F. Peyrard, D. Lavielle, and C. Chatry, "FASTRAD: A 3D CAD Interface for Radiation Calculation and Shielding”, $11^{\text {th }}$ International Conference on Radiation Shielding (ICRS-11), April 13-18, 2008, Callaway Gardens, Pine Mountain, Georgia, USA.

[14] "FASTRAD Software - The 3D CAD Tool For Radiation Shielding Analysis", TRAD [Online]. Available: https://www.fastrad.net/.

[15] G. Santin, "Normalisation modelling sources", Geant4 [Online]. Available: http://geant4.in2p3.fr/2007/prog/GiovanniSantin/GSantin _Geant4_Paris07_Normalisation_v07.pdf.

[16] J. D. Sullivan, "Geometric factor and directional response of single and multi-element particle telescopes', Nuclear Instruments and Methods”, vol. 95, no. 1, pp. 5-11, Aug. 1971.

[17] TRAD, "SAC-D CARMEN - Analyse sectorielle", Sep. 2012.

[18] F. Bezerra et al., "CARMEN/MEX test board for the study of radiation effects on electronic components aboard JASON-2 and SAC-D satellites", $9^{\text {th }}$ European Conference on Radiation and Its Effects on Components and Systems, RADECS 2007, 10-14 Sept. 2007.

[19] S. Bourdarie, P. O’Brien, D. Boscher, and T. Guild, "The International Radiation Belt Environment Modeling (IRBEM) library”, 2017 [Online]. Available: https://sourceforge.net/p/irbem/.

[20] P. Valot, and J. Engelmann, "Pitch Angle Distribution of Geomagnetically Trapped Protons for $1.2<\mathrm{L}<2.1$," Space Research XIII, Akademic Verlag, Berlin, 1973.

[21] J. G. Roederer, "Dynamics of Geomagnetically Trapped Radiation”. Springer, 1970.

[22] M. Kruglanski and J. Lemaire, "Trapped Proton Anisotropy at Low Altitudes”, Tech. Note 6, TREND-3 Report, Apr. 1996.

[23] M. Siegl, 'Standard radiation environment monitor: simulation and inner belt flux anisotropy investigation', M. S. thesis, Lulea Univ. Technol., Kiruna, Sweden, 2009.

[24] D. Heynderickx, M. Kruglanski, V. Pierrard, J. Lemaire, M. D. Looper, and J. B. Blake, "A low altitude trapped proton model for solar minimum conditions based on SAMPEX/PET data,” IEEE Trans. Nucl. Sci., vol. 46, pp. 1475-1480, Dec. 1999. 